\title{
Analysis on Depression Among LGBTQ Population
}

\author{
Wei Huang ${ }^{1}$ \\ ${ }^{I}$ Department of Psychology, University of California, Irvine, CA 92617, USA \\ *Corresponding author. Email:huangw12@uci.edu
}

\begin{abstract}
This study explores the current status and tendency of the LGBTQ population, who are suffering from depression, as well as measures to release their stress. Public opinion gives homosexuals more pressure and they are prone to suffer from depression. There is insufficient research on depression in marginalized groups such as the LGBTQ population. The study supplements the research about the depression tendency of the LGBTQ communities and proposes methods to improve it. The author registered a non-governmental organization, which provides psychological to the LGBTQ population under the national health system. The research subjects are selected from 41 homosexuals from April 2020 to August 2020, and 40 normal people as the control group. The Hamilton Depression Scale is used to test the depression and tendency of the subjects. The background information and test results of the research subjects are analyzed, providing psychological counseling to homosexuals. It is found that the LGBGQ population has a higher tendency for depression. In addition, the causes and treatment are discussed in the article. The acceptance of family and social pressure are potential risk factors for depression among the LGBTQ population.
\end{abstract}

Keywords: LGBTQ, Depression, Mental Health

\section{INTRODUCTION}

Due to historical and cultural factors, the Chinese people's acceptance of marginal groups such as the LGBTQ population has not gradually increased with economic development and the opening up of the country. Because of the influence of the older generation's perceptions, insufficient exposure to LGBTQ groups, and the spread of some negative news, many people have prejudices against the LGBTQ population, which brings great pressure to the group, decreases their quality of life, and even leads to tragedies such as suicide. The research on the mental health problems of the LGBTQ population is not sufficient and it is necessary to study the cause and treatment of it. At present, the LGBTQ community in China has a population of approximately 70 million, but only $5 \%$ of the LGBTQ population determine to disclose their sexual orientation. In particular, $70 \%$ of gays and lesbians choose to marry heterosexuals to hide their real sexual orientation, which is called "sham marriage" [1]. The LGBTQ population is under great pressure in life and work and they are more likely to suffer from mental illnesses, such as anxiety disorder and depression. Therefore, the author will investigate the cause and treatment of depression of the LGBTQ population. It is expected that this research will help LGBTQ group relieve the mental stress and improve their quality of life.

\section{OVERVIEW}

\subsection{Background}

LGBTQ stands for lesbian, gay, bisexual, transgender, and queer. The LGBTQ population is considered as sexual minorities, and their sexual identity and orientation is different from the mainstream society [3]. According to statistics, $16 \%$ of LGBTQ youth and $35 \%$ of transgender people attempt to suicide; while $3.2 \%$ of the general population report that they have committed suicide in the past year. It is reported that $18 \%$ of the LGBTQ population have experienced verbal abuse and $37 \%$ of those experienced physical abuse. The LGBTQ population are as twice as possible to have psychological problems than the general population [8].

With the acceleration of the pace of life and the increase of pressure, depression has become a common mental disorder. Many people suffer from mild depression or even severe depression because of stress, anxiety, and other factors. Severe depression may develop into other psychological disorders like bipolar disorder, which may seriously affect the quality of life of 
patients. The LGBTQ population is more likely to develop a sense of helplessness and depression than other people as the LGBTQ group is easily rejected by their families, friends, and other people in society. Homosexualities are more likely to be prejudiced by people around in the environment and report suffering from anxiety. What is more, the possibility of drug abuse among the LGBTQ community is higher. These factors lead to the vulnerability to depression and anxiety disorders in the LGBTQ community.

In China, the situation of the LGBTQ population is not same as in other countries. In Western countries, people's lack of understanding and discrimination towards the LGBTQ population has been changing to acceptance and tolerance. However, in China, due to the Chinese traditional culture and the ambiguity of current laws, the living conditions of most LGBTQ people are not good, and their basic rights cannot be effectively protected. LGBTQ population is discriminated against in the workplace, and many LGBTQ individuals choose not to disclose their sexual orientation and gender identity. In terms of education, LGBTQ students are more likely to be bullied on campus. These environmental and cultural factors make Chinese LGBTQ communities face discrimination and prejudice. As a result, they suffer from more stress and are more prone to depression.

In China, about 40 percent of the general population adopt a conservative and relative negative attitude on the LGBTQ population. About 15 percent of middle-aged and senior people, especially those live in rural and remote areas, perceive homosexual orientation as a disease and LGBTQ individuals as abnormalities and aliens, and cannot accept the LGBTQ population. The main reasons are that the LGBTQ population is the destruction of social ethic in the traditional Chinese fertility and they disrupt the social order and the heterosexual marriage system. Moreover, many people believe that they are conducive to the spread of many diseases such as AIDS. Social prejudice also affects the LGBTQ communities in China and makes them more susceptible to anxiety disorder and depression.

\subsection{About the Research}

The author registered a non-governmental organization in Beijing, which provides psychological assistance and support services to homosexual groups under the national health system. The selected subjects of the research were 41 homosexuals and 20 normal people, who received support in the organization from April 2020 to August 2020 after getting their agreement to do the investigation. 31 subjects are male and 30 subjects are female, ranging from 18 years old to 43 years old.

The author analyzed the general background of the subjects and the test scores for the scale. The Hamilton Depression Scale is used to test the depression tendency of subjects in the study. It is a scale which is commonly used clinically to test whether a subject has depression or a tendency to have depression. It is efficient and simple for subjects to assert for depression and depression tendency [4].
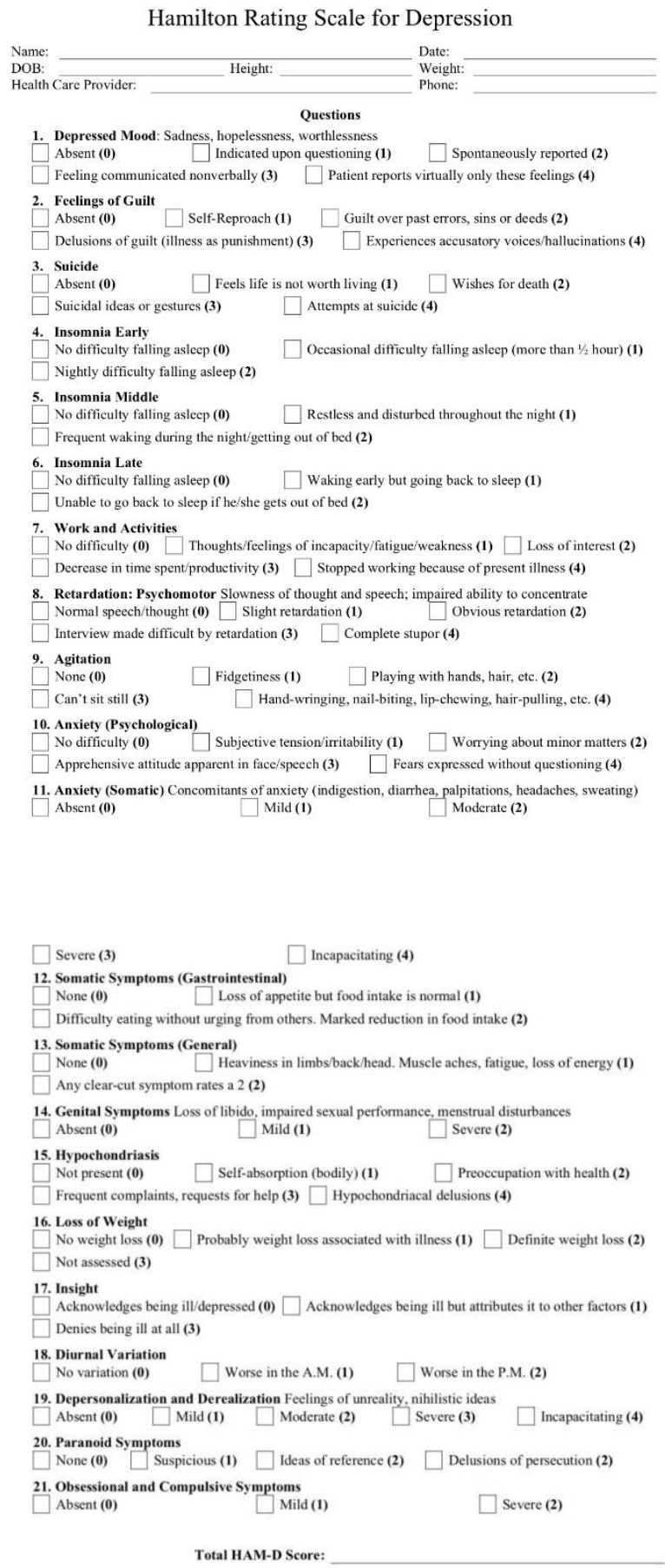

Figure 1 Hamilton Depression Scale for Depression

\section{DATA ANALYSIS}

The results are divided into four levels: the first level indicates the total score is less than 7 points, and the 
subject does not suffer from depression; the second level is with the total score ranging from 7-17, and the subject have mild depression; the third level represents the total score in 17-24 points, and the subject must have moderate depression; the fourth level means the total score is higher than 24 points, and the subject has severe depression.

Among 40 homosexual subjects, a total of eighteen people scored between 8-16 after the test and might suffer from depression. Nine individuals scored around 17 and suffered from mild depression. Thirteen individuals scored less than 7 points with no depression. Among the 40 cases in the control group, 12 individuals scored between 7-17 and might suffer from depression. One scored 18 and suffered from mild depression. The remaining 28 individuals scored below 7 points and did not suffer from depression. Among the homosexual groups being surveyed, the rate of depression tendency and depression is higher than that of common people. The test scores of the subjects and control group for Hamilton Depression Rating Scale are summarized in figure 2 .

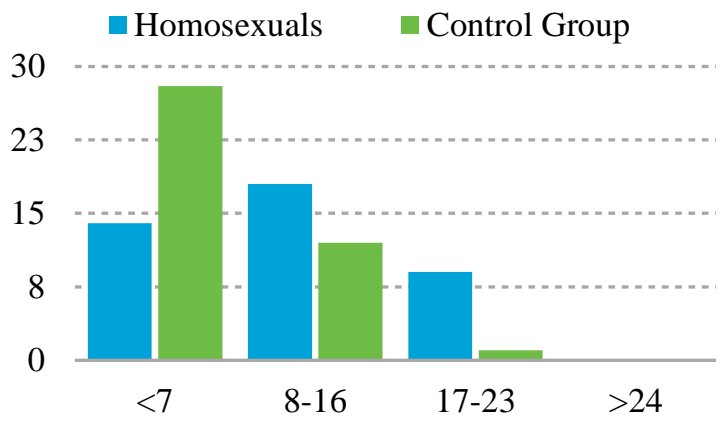

Figure 2 Test Scores of Hamilton Depression Rating Scale

\section{DISCUSSION}

\subsection{Causes}

The depression tendency is relatively high among the LGBTQ population and it is caused by discrimination against the LGBTQ population, low self-esteem, and other factors, like malevolence and stress from the families and society. The author investigates the age of the subjects and whether their family members accept their sexual orientation. It is found that the age and family acceptance of the LGBTQ population influence the tendency and severity of their depression. Family support helps improve the resilience of depression and stress for the LGBTQ population. The homosexual subjects, who are not supported by the family or the public, are more prone to suffer from anxiety and depression.

A potential risk factor for depression is microaggression which is likely to be ignored by researchers and even the LGBTQ population themselves. Microaggression refers to the everyday indignities like negative attitudes towards marginalized groups. It is intentional or unintentional biased behaviors which can make victims feel insulted [2]. The daily and long-term prejudice and micro-aggression lead to chronic stress and anxiety on the LGBTQ population. According to the minor stress theory, the chronic stress and the hostile environment with micro-aggression play a vital role in the disparities of mental health for the LGBTQ population [9].

Moreover, $90 \%$ of subjects said that they were bullied or assaulted in school or workplace due to their sexual orientation. The odds of experiencing bullying for the LGBTQ population is as five times as that of their heterosexual peers, which may cause them to suffer from depression [4]. The bulling of other people around the LGBTQ population negatively affects their self-esteem and low self-esteem is a rick factor for the depression. The low self-esteem also leads to negative thoughts and perception of the world. The long- term bullying would further cause the sense of helplessness and suicidal behavior. $55 \%$ of subjects also report that they have attempted to commit suicide because they have a sense of hopelessness.

\subsection{Treatments}

Compared with the normal control group, the LGBTQ population is more likely to suffer from anxiety and depression with more serious degree. The pressure on the LGBTQ population is relatively larger, which is mostly because of the prejudice of society and family pressure. Family support and acceptance help release the pressure of the LGBTQ population. Subjects who are accepted by their families are more resilient to the depression and show a milder tendency of depression. Family support is proved to be efficient to help the LGBTQ population release pressure and cure depression [7].

A positive and friendly environment helps reduce the depression tendency of the LGBTQ population. According to the minority stress theory, the acceptation and positive attitude of society are associated with the lower tendency of depression [8]. The public opinion on the LGBTQ population is relatively negative because the history and culture in China shape people's acceptance and attitudes towards the LGBTQ communities. As the environment factor plays a vital role in the depression tendency of the LGBTQ population, the public opinion can be positively directed to reduce prejudice to help solve this problem.

In addition, an efficient therapy for depression is the cognitive behavioral therapy (CBT). Cognitive behavioral therapy is based on the psychological cognitive theory that negative feelings lead people to have distorted thoughts and increase the depression tendency [5]. It is helpful for the LGBTQ population to manage their depression and anxiety, reconstructing their ways to perceive stressors around them. The cognitive behavioral therapy helps the LGBTQ population focus on 
their negative thoughts and view them in a different and positive way. By teaching them stress management skills, therapists could help the LGBTQ population reduce the symptoms of depression.

\section{CONCLUSION}

The high rate of anxiety and depression among homosexuals is a complex process, which is affected by multiple factors such as family pressure, age, and public opinion in the external environment. For homosexual patients suffering from anxiety and depression, appropriate psychological counseling like CBT can effectively relieve their depressive symptoms. The understanding of family members and friendly environment can also help the LGBTQ communities deal with stress and anxiety. This study analyzes the results of the Hamilton Scale taken by the homosexual group and control group. Compared with subjects of the control group, the average score of the homosexual group is higher and the tendency to suffer from depression is also higher. Through psychological counseling and analysis of background information, the author further reveals the effect of family factors, attitudes, and prejudices of the external environment on the anxiety and depression of the LGBTQ population and their mental health. Positive public opinion, appropriate psychological counseling, and family support are of great significance to eliminate the prejudice against the LGBTQ population and improve the living environment and quality of the LGBTQ communities. However, the sample size of this study is not large enough. Only the self-report method is adopted when investigating the family background and age information of subjects, and there may be potential bias when making conclusion. In the future, more experiments and case studies are needed to research the psychological problems faced by the LGBTQ population.

\section{AUTHORS' CONTRIBUTIONS}

Wei Huang did the investigation and analyzed the results, finishing the paper by herself.

\section{ACKNOWLEDGMENTS}

I would like to give thanks to my family and friends for their support. Without their assistance, the work cannot be completed. I am grateful for all of the subjects participated in this project and provide information to help the research. I am particularly indebted to Professor Sznecs for inspiration and assistance.

\section{REFERENCES}

[1] Australian Bureau of Statistics National Survey of Mental Health and Wellbeing: Summary of Results, 2007, 4326.0. Australian Government, Canberra.
[2] J. Desmond-Harris, "What Exactly Is a Microaggression?" Vox, 16 Feb. 2015, www.vox.com/2015/2/16/8031073/what-aremicroaggressions.

[3] D. Higa, M. J. Hoppe, T. Lindhorst, S. Mincer, B. Beadnell, D. M. Morrison, E. A. Wells, A. Todd, \& S. Mountz, Negative and Positive Factors Associated With the Well-Being of Lesbian, Gay, Bisexual, Transgender, Queer, and Questioning (LGBTQ) Youth. Youth \& Society, 2012, 46(5), 663-687.

https://doi.org/10.1177/0044118x12449630

[4] H. Elizabeth, Medpagetoday.com. 2020. Bullying Preceded Many LGBTQ Youth Suicides. [Online] Available

at: https://www.medpagetoday.com/psychiatry/general psychiatry/86682 [Accessed on 15 December, 2020].

[5] H. Jill, et al. Abnormal Psychology (17th Edition). 17th ed., Pearson, 2016.

[6] M. Hamilton, 2020. A RATING SCALE FOR DEPRESSION. [Online] Available at: https://www.ncbi.nlm.nih.gov/pmc/articles/PMC49 $5331 /$ ?page $=1$ [Accessed on 15 December, 2020].

[7] H. Treniece Lewis. "Cultural Orientation, Family Cohesion, and Family Support in Suicide Ideation and Depression among African American College Students." Wiley Online Library, 1 Dec. 2000, onlinelibrary.wiley.com/doi/abs/10.1111/j.1943278X.2000.tb01100.x.

[8] P. Marian \& S. Anthony \& M., Anne \& P. Sunil, PRIVATE LIVES A report on the health and wellbeing of GLBTI Australians, 2006.

[9] Woodford, R. Michael, et al. "Depression and Attempted Suicide among LGBTQ College Students: Fostering Resilience to the Effects of Heterosexism and Cisgenderism on Campus." Journal of College Student Development, vol. 59, no. 4, 2018, pp. 421-38. Crossref, doi:10.1353/csd.2018.0040. 